2009-01-01

\title{
Electronic Tunability of Ferroelectric Liquid Crystal Infiltrated Photonic Crystal Fibre
}

\author{
Sunish Mathews \\ Technological University Dublin \\ Yuliya Semenova \\ Technological University Dublin, yuliya.semenova@tudublin.ie \\ Gerald Farrell \\ Technological University Dublin, gerald.farrell@tudublin.ie
}

Follow this and additional works at: https://arrow.tudublin.ie/engscheceart

Part of the Electrical and Computer Engineering Commons

\section{Recommended Citation \\ Mathews, S., Semenova, Y. \& Farrell, G.(2009) Electronic Tunability of Ferroelectric Liquid Crystal Infiltrated Photonic Crystal Fibre. Electronic Letters, Vol.27, no.13, pp.2482-2488. doi:10.1049/ el.2009.0580}

This Article is brought to you for free and open access by the School of Electrical and Electronic Engineering at ARROW@TU Dublin. It has been accepted for inclusion in Articles by an authorized administrator of ARROW@TU Dublin. For more information, please contact arrow.admin@tudublin.ie, aisling.coyne@tudublin.ie, gerard.connolly@tudublin.ie.

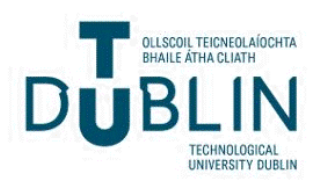




\title{
Electronic Tunability of Ferroelectric Liquid Crystal Infiltrated Photonic Crystal Fibre
}

\author{
Sunish Mathews, Yuliya Semenova, Gerald Farrell
}

The electronic tunability of ferroelectric liquid crystal filled photonic crystal fibres is demonstrated for the first time. The tunability is obtained in the wavelength range of $1500-1600 \mathrm{~nm}$, with a free spectral range of $\sim 20 \mathrm{~nm}$. The electronic tunability is achieved by applying an electric field onto the infiltrated photonic crystal fibre which facilitates the photonic bandgap tuning owing to the microsecond order switching speeds of Ferroelectric Liquid crystals. The polarized transmission spectrum shows the behavior of a tunable notch filter with a change in applied voltage. The results obtained could pave the way for the fabrication of fast tunable all-in-fibre devices in the telecom wavelength range.

Introduction: Photonic crystal fibres (PCFs) infiltrated with Liquid Crystal materials have emerged as a new class of optical fibres commonly called Photonic Liquid Crystal Fibres (PLCFs) [1]. These have revealed many interesting properties having potential applications in all-optical modulation, fibre-optic sensing and have been projected as a promising candidate for the fabrication of in-fibre tunable devices. The importance of all-in-fibre devices arises from the fact that, such devices are fully compatible with existing fibre technology and being tunable, they can be adjusted to specific system requirements. Furthermore, with in-fibre tunable devices the shortcomings of 
their bulk counterparts such as the losses due to excessive optical elements, complexity of optical coupling etc can be eliminated or minimized. Infiltration of liquid crystals into the air-holes of PCFs makes them highly sensitive to field variations allowing for temperature or electrical tuning of the propagation properties. Temperature and electronic tuning of different types of PCFs infiltrated with Nematic Liquid Crystals based on photonic bandgap (PGB) tuning have been demonstrated previously [2 - 4]. Nematic liquid crystals are characterized by relatively slower tuning speeds $(\sim$ milliseconds $)$ and furthermore, thermal tuning can be employed for applications where smooth and slow changes are required. By comparison to nematic liquid crystals, Ferroelectric Liquid Crystals (FLC) possess fast switching speeds, excellent electro-optic response, larger switchable birefringences and better phasestability upon repeated electronic switching [5]. In this letter we demonstrate for the first time, the electronic tunability of a FLC infiltrated PCF, in the wavelength range of $1500-1600 \mathrm{~nm}$. A tunable range of $\sim 20 \mathrm{~nm}$ is achieved and a linear response of the spectral shift with a change in voltage is observed for voltages from $60 \mathrm{~V}-110 \mathrm{~V} \mathrm{DC}$.

Theory and Experimental Setup: After infiltration of the FLC material, which has a higher refractive index than silica, into the cladding region of a solid core $\mathrm{PCF}$, the cladding region assumes an effective refractive index higher than the core and the wavelength-dependent guidance of light in this case is due to the photonic bandgap mechanism [6]. The application of an electric field onto the PLCF induces FLC molecular director reorientation in the infiltrated cladding region, which changes the effective refractive index of the 
cladding, thereby facilitating Photonic Bandgap tuning of the PCF crosssection.

The ferroelectric liquid crystal material used for our experiments is FELIX 019/000 (AZ Electronics), which has a Smectic C phase in the temperature range from $2-60{ }^{\circ} \mathrm{C}$, with a tilt angle of $\sim 38^{\circ}$. The ordinary $\left(\mathrm{n}_{\mathrm{o}}\right)$ and extraordinary $\left(n_{e}\right)$ refractive indices of the material are 1.487 and 1.651 respectively, measured at $30^{\circ} \mathrm{C}$ at $589.3 \mathrm{~nm}$. Our experiments were performed at room temperature. The FLC was infiltrated into a Polarization Maintaining Photonic Crystal Fibre (PMPCF), PM-1550-01 (Crystal Fibre A/S) with a solid silica core. The PCF has two large holes of diameter $4.5 \mu \mathrm{m}$ and small holes of diameter $2.2 \mu \mathrm{m}$ with a pitch $\Lambda$ (inter-hole spacing) of $4.4 \mu \mathrm{m}$. The infiltration was performed by dipping the cleaved end of the fibre into the FLC material at a temperature between $80-90{ }^{\circ} \mathrm{C}$. The FLC molecules get drawn into the PCF, due to capillary forces. The PCF section used for this study had a length of $5 \mathrm{~cm}$, which was placed between a pair of electrodes to allow the application of an electric field perpendicular to the fibre axis. The experimental setup is as shown in fig. 1. Light from the broadband source (Superlum Diodes Ltd.; 1490-1640 nm) was coupled into a fibre linear polarizer and then coupled into the infiltrated end of the PLCF. The PLCF output coupled through a free-space analyzer (infrared polarizer) is inputted to an Optical Spectrum Analyzer. The coupling of light at the input and collection at the output from the PLCF was done using the 3-stage XYZ-nano-positioner waveguide stage. The coupling loss was circa $1.5-2.0 \mathrm{~dB}$ with fibre buttcoupling using the nano-positioner. The polarized transmission spectrum was recorded for a voltage range from $0-150 \mathrm{~V} D C$. 
Results: Initial studies on the PLCFs based on polarization microscopy show $\sim 1.3 \mathrm{~cm}$ of infiltration into the PCF with the alignment being largely along the axis of the fibre. The polarized (parallel) transmission spectrum obtained for different voltages showed transmission minima (notch) at around $1550 \mathrm{~nm}$, which is a characteristic of PBG guidance in PCFs [6]. No change in the transmission spectrum was observed for voltages from $0 \mathrm{~V}-60 \mathrm{VDC}$, suggesting that at these voltages the reorientation of the FLC molecules does not take place and the propagation is governed by the ordinary refractive index of the FLC within the holes of the PCF. For voltages from $60 \mathrm{~V}$ to $110 \mathrm{~V}$ DC, a shift of the transmission minima towards the lower wavelengths is observed with an increase in voltage, as shown in Fig. 2. An overall shift of $\sim$ $22 \mathrm{~nm}$ from $1554 \mathrm{~nm}(60 \mathrm{~V})$ to $1532 \mathrm{~nm}(110 \mathrm{~V})$ was observed. The spectral profile shows the features of a notch-type filter which could be tuned with the application of the electric field. The shift of the transmission notch for the voltage range from $60 \mathrm{~V}-110 \mathrm{~V}$ shows a linear response, as shown in Fig. 3. The reorientation effect of the FLC molecules takes place above $\sim 60 \mathrm{~V}$ and at these voltages the PBG positions depend on the FLC extraordinary refractive index. The electrically induced molecular tilt of FLC molecules not only changes the effective refractive index of the cladding, but also creates anisotropy in the PLCF cross section. Further increases in the voltage to 150 V DC resulted in no shift in the spectrum, with the transmission minima remaining centered at $1532 \mathrm{~nm}$. This could be attributed to the fact that the FLC material may have attained the maximum tilt angle and at these voltages further reorientation does not take place with an increase in voltage. An all-in- 
fibre tunable notch filter of this type, in this particular wavelength range, would find applications in spectral shaping of the cavity of a modelocked fibre laser, in tunable dispersion control within and outside the cavity and also for Amplified Spontaneous Emission (ASE) filtering and gain flattening of erbium doped fibre amplifiers.

Conclusion: The electronic tunability of Ferroelectric Liquid Crystal infiltrated Photonic Crystal Fibre is demonstrated in the wavelength range of $1500 \mathrm{~nm}$ $1600 \mathrm{~nm}$ with a tunable range of $\sim 20 \mathrm{~nm}$. The transmission profile of the PLCF shows a tunable notch-filter like behaviour in the wavelength range from $1532 \mathrm{~nm}-1554 \mathrm{~nm}$ and the shift in the transmission minima with change in the applied voltage is found to be linear. A device based on such a PLCF would find application in the fabrication of an all-in-fibre tunable notch filter for the spectral shaping of the cavity for fibre lasers and for the ASE filtering of fibre amplifiers.

\section{References}

1. WOLINSKI T.R., ERTMAN S., LESIAK P., DOMANSKI A.W., CZAPLA A., DABROWSKI R., NOWINOWSKI-KRUSZELNICKI E. WOJCIK J., 'Photonic liquid crystal fibres - a new challenge for fibre optics and liquid crystal photonics', Opto-Elect. Rev., 14 (4), 329, (2006).

2. DU F., LU Y. Q, WU S. T., 'Electrical tunable liquid-crystal photonic crystal fibre', App. Phys. Lett. 85, (12), 2181, (2004).

3. LARSON T., BJARKLEV A., HERMANN D., BROENG J., 'Optical Devices based on liquid crystal photonic bandgap fibres', Opt. Exp. 11 (20), 2589, 2003.

4. ERTMAN S., CZAPLA A., NOWECKA K., LESIAK P., DOMANSKI A.W., WOLINSKI T.R., DABROWSKI R., 'Tunable highly-birefringent Photonic Liquid Crystal Fibres', Proc. Instrumentation and measurement Technology Conference - IMTC 2007, Warsaw, Poland, May 1-3, 2007 
5. HARTMAN N.F., GAYLORD T.K., DRABIK T.J., HANDSCHY M.A., 'Phase stability of ferroelectric liquid crystals upon repeated switching and static temperature characteristics', App. Opt., 32 (20), 3720, (1993).

6. LITCHINITSER N.M., DUNN S.C, STEINVURZEL P.E, EGGLETON B.J, WHITE T.P., MCPHEDRAN R.C, DE STRERKE C.M, "Application of an ARROW model for designing tunable photonic devices", Opt. Exp., 12, 8, 1540 (2004).

\section{Authors' affiliations:}

Sunish Mathews, Yuliya Semenova, Gerald Farrell (Photonics Research Centre, School of Electronics and Communications Engineering, Dublin Institute of Technology, Dublin 8, Ireland)

E-mail : sunish.mathews@dit.ie 


\section{Figure captions:}

Figure.1 Schematic diagram of the experimental setup for studying the electronic tunability of FLC infiltrated PCF.

Figure.2 Polarized Transmission spectrum of the FELIX 019/000 infiltrated PMPCF at different voltages from $60 \mathrm{~V}-110 \mathrm{~V} \mathrm{DC}$.

Figure.3 Electronic tunability of the spectral minima (notch) of the PLCF. 
Figure. 1

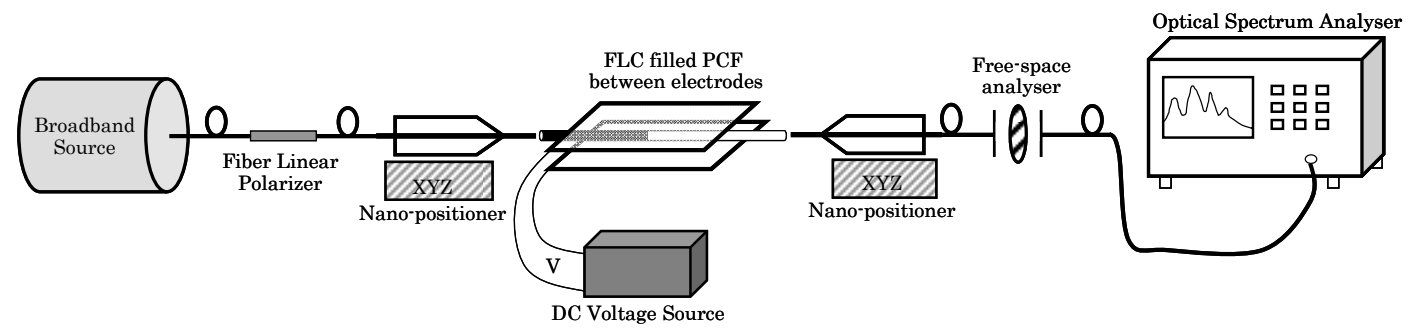


Figure. 2

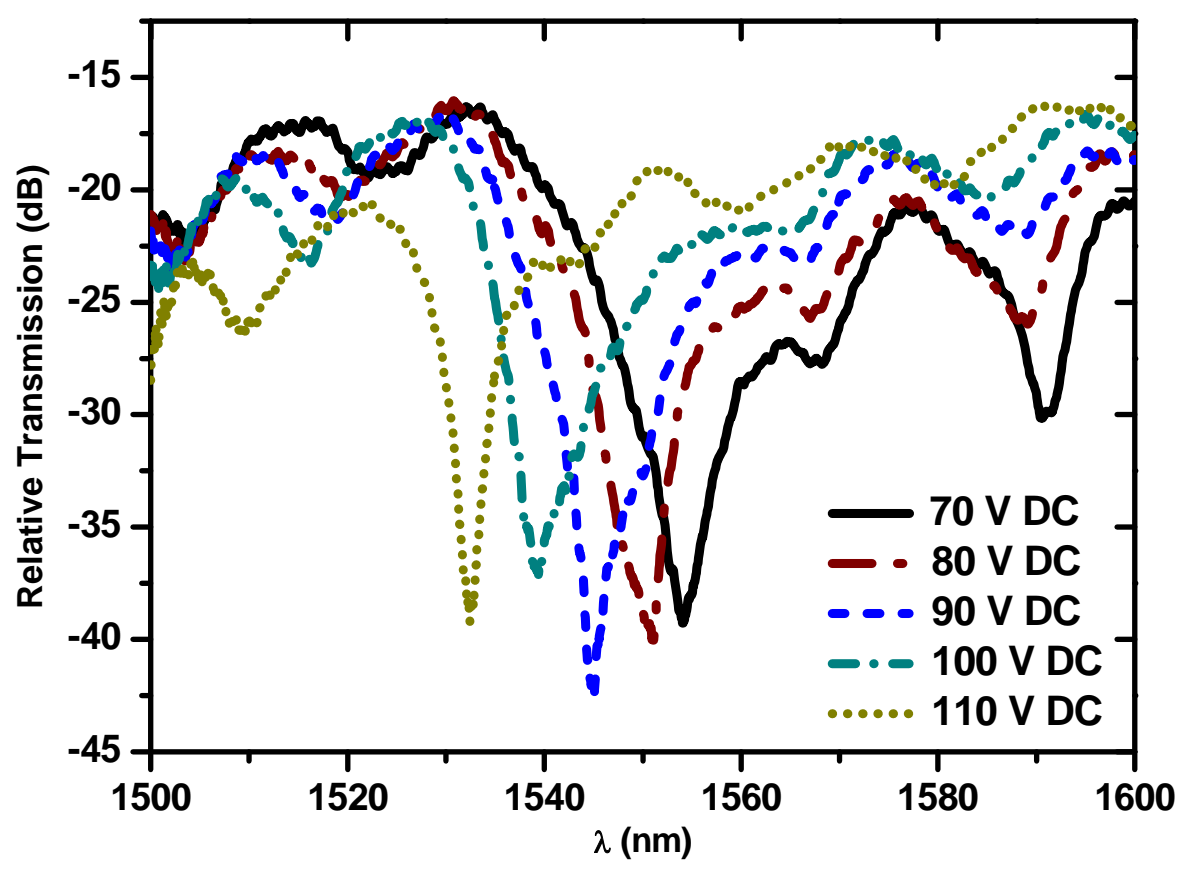


Figure. 3

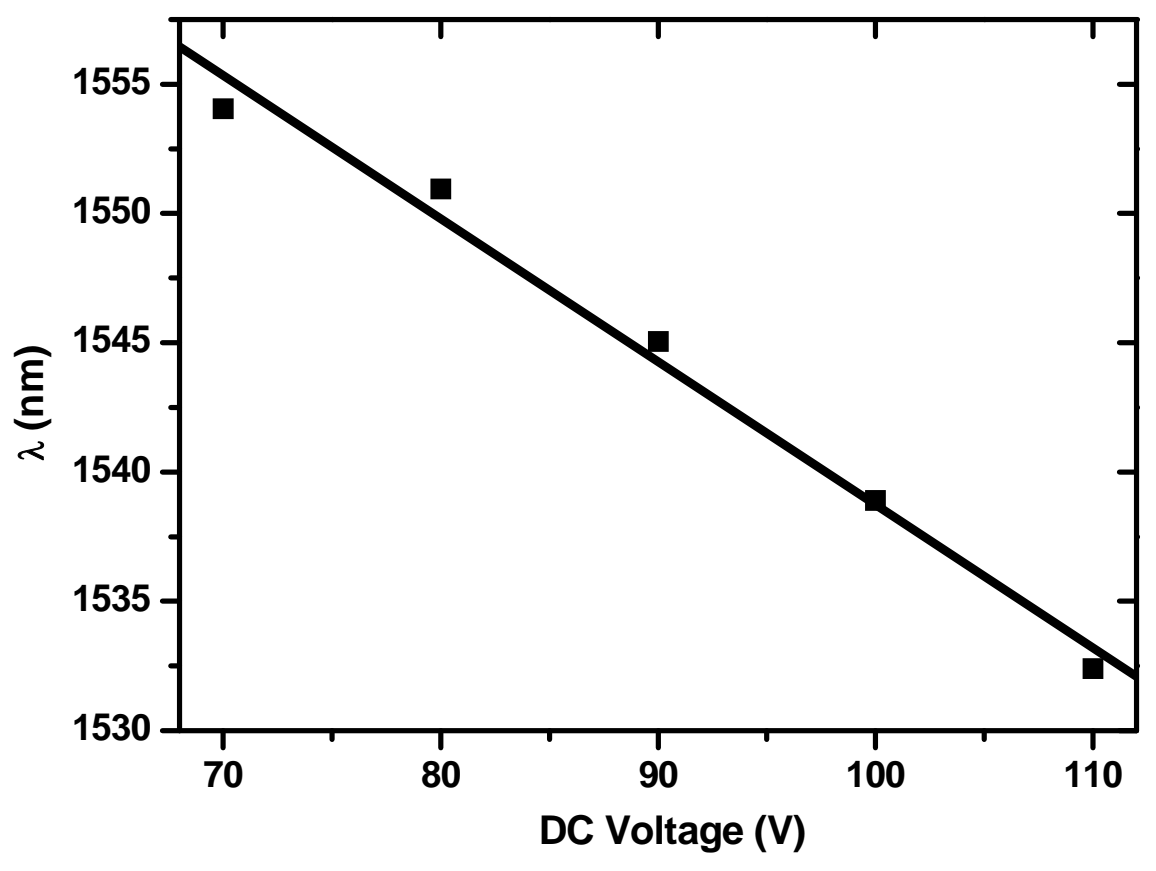

